\title{
The Effects of Obstacles and Age on Walking Time within a Course and on a Balance Beam in Preschool Boys
}

\author{
Kosho Kasuga $^{1}$, Shin-ichi Demura ${ }^{2}$, Hiroki Aoki ${ }^{3}$, Toshiro Sato ${ }^{4}$, Sohee Shin ${ }^{1}$, Haruka Kawabata ${ }^{2}$ \\ ${ }^{1}$ Gifu University, Gifu, Japan \\ ${ }^{2}$ Graduate School of Natural Science \& Technology, Kanazawa University, Kanazawa, Japan \\ ${ }^{3}$ Kanazawa College of Art, Kanazawa, Japan \\ ${ }^{4}$ Niigata University of Health and Welfare, Niigata, Japan \\ Email: aoki@kanazawa-bidai.ac.jp
}

Received March 26 ${ }^{\text {th }}$, 2012; revised April 27 $7^{\text {th }}$ 2012; accepted May $7^{\text {th }}, 2012$

\begin{abstract}
This study aimed to examine the effect of obstacles and age on walking time within a course $(10 \mathrm{~cm}$ width $)$ and on a balance beam in 158 preschool boys, aged 4 (47), 5 (50), and 6 (61) years. An obstacle 5 or 10 $\mathrm{cm}$ in height (depth, $11.5 \mathrm{~cm}$ and width, $23.5 \mathrm{~cm}$ ) was placed at the halfway point of the course drawn on the floor and on the balance beam (200 cm in length, $10 \mathrm{~cm}$ in width and $30 \mathrm{~cm}$ in height). Children walked to the end and returned to the original position as fast as possible within the above course and on the balance beam under three conditions: low, and high, and no obstacle. Walking time was measured for each condition. Subjects in all age groups were able to walk within a course significantly faster than on the balance beam; in the former test, boys aged 4 to 4.5 years were slower than boys aged 5.5 to 6 years as well as the 6-year-olds, and boys aged 4.5 to 5 years were slower than 6-year-olds; in the latter test, the boys aged 4 to 4.5 years were slower than boys aged 4.5 to 5 years, 5 years and 6 years, the boys aged 4.5 to 5 years were slower than 5-year-olds and 6-year-old boys, and the 5-year-old boys were slower than the 6-year-olds. Walking times under all obstacle conditions were longer in 4-year-old boys than in the 5 and 6 -year-old boys, longer in boys aged 5 to 5.5 years than in the 6-year-olds, and longer in boys aged 5.5 to 6 years than in the 6-year-olds. Walking times within the course was shorter than those on the balance beam under all obstacle conditions. In addition, walking times both in the course and on the balance beam were shorter in the following order: no obstacle (shortest); low obstacle; high obstacle (longest). In conclusion, in-course walking is faster than balance beam walking regardless of age or the presence of an obstacle. Furthermore, improvements in the ability to complete both of these walking tasks may differ with age.
\end{abstract}

Keywords: Dynamic Balance; Boys; Balance Beam

\section{Introduction}

It is important to adequately evaluate the developmental process of nerve function because the neurological system develops significantly during the preschool period. Nerve function is related to coordination, and balance ability is an important component of coordination. Balance ability is divided largely into static balance and dynamic balance abilities, with the latter being the ability to maintain postural stability during various movements (Aoki et al., 2011). Preschool children learn to perform various locomotive movements with age. Dynamic balance contributes greatly to achievement of these movements. Hence, it is vital to adequately evaluate the development of dynamic balance in the preschool period.

Line and balance beam walking tests have been designed to evaluate the dynamic balance of preschool children (Demura, 1995; Chandler et al., 1996; Bürgi et al., 2011). Since the former test is very easy, most older children can achieve it within a certain time (ceiling effect). Meanwhile, the latter test creates problems where younger children can fall off the beam and be injured, or are unable to perform according to true ability due to fear of falling (Kasuga et al., in press). In a recent attempt to evaluate the dynamic balance characteristics of preschool children, the ability to walk on a balance beam in the presence of an obstacle was examined. As stepping over the obstacle while walking requires compound movements, this task is more difficult to perform than merely walking in a straight line or walking on a balance beam. Additionally, stepping over an obstacle while walking on the balance beam is more difficult than simply walking on a level surface. In short, it is assumed that although both in-course and balance beam walking become more difficult when an obstacle is included, the delayed time is larger in the latter.

Many researchers have studied the development of dynamic balance of preschool children (Demura, 1993; Demura, 1995; Chandler et al., 1996; Bürgi et al., 2011). Demura et al., (1994) reported that line walking time did differ with respect to age in 3 to 5-year-old children, but was shorter for 6-year-old children in comparison to 5-year-old children. According to Kasuga et al., (in press), children aged 6.5 to 7 years showed a difference in course walking time with obstacles as compared to children aged 5 to 5.5 years, but not compared to children aged 5.5 to 6 years.

In addition, Aoki et al. (2011) reported that, regardless of whether or not an obstacle was present, walking time on the balance beam was significantly longer for 5-year-old children than for children aged 6.5 to 7 years. In short, although a difference of test methods also affects walking time, results in previous studies are inconsistent. Developmental trends with respect to age may differ between the within-course walking and balance beam walking tasks, with or without the presence 
of an obstacle.

The ability to walk begins at about one year of age, and individual differences in ability disappear at about 6 years old. Even though it employs the same basic walking skills, walking on a balance beam has high difficulty and even children who have mastered level walking find it hard to complete. When stepping over an obstacle is added, the degree of difficulty increases even further (double tasks). As the difficulty of an exercise task increases, exertion of dynamic balance is also required for its achievement. In this study, beneficial insight may be found on the development across age and gender of performance of exercise tasks based on walking with different difficulty levels.

This study aims to examine the effects of obstacle presence and age on walking time within a course and on a balance beam.

\section{Methods}

\section{Subjects}

The subjects included 158 healthy boys aged 4 to 6 years. Table 1 shows their physical details. The experimental purpose and methods were explained to all preschool children and their parents, and their consent was obtained. The present protocol was approved by the Kanazawa University Department of Education.

\section{Procedures}

Walking in-course: obstacles (depth, $11.5 \mathrm{~cm}$ and width, 23.5 $\mathrm{cm}$ ) with different heights $(5 \mathrm{~cm}$ and $10 \mathrm{~cm}$ ) were set at the halfway point of a course $(10 \mathrm{~cm}$ width and $200 \mathrm{~cm}$ length) (see Figure 1). The subjects walked within the course under three conditions: no obstacle, low obstacle, and high obstacle. The time was measured from when the subjects crossed the starting line (line tape length, $10 \mathrm{~cm}$ and width, $10 \mathrm{~cm}$ ), reached a turn line, to when they returned to the starting line.

Walking on the balance beam: The same obstacle was set at the halfway point of the balance beam $(10 \mathrm{~cm}$ width, $30 \mathrm{~cm}$ height, $200 \mathrm{~cm}$ length) (see Figure 1). The subjects walked on the beam under three conditions: no obstacle, low obstacle, and high obstacle. The time was measured from when the subjects crossed the starting line, reached a turn line, to when they returned to the starting line.

Children were instructed to walk as fast as possible, to change direction quickly after reaching the turn line, and to return to the original position. Subjects carried out three trials for each test. We repeated the measurement if, while walking, they fell or their foot touched the obstacle. In addition, if the above occurred three times in succession, measurement was judged to be impossible for the subject.

\section{Data Analysis}

The intra-class correlation coefficient (ICC) for each test was calculated to examine trial-to-trial reliability.

Three-way ANOVA was used to ascertain the mean differences of each test, age, and obstacle condition for each walking time. When a significant interaction or main effect was observed, a Tukey's Honestly Significant Difference (HSD) test was used for multiple comparisons. A linear or curve regression was calculated to examine the relationship between each walking time and the subject's age, and the significance of the above
Table 1.

Basic statistics of age, height and weight.

\begin{tabular}{lccccccc}
\hline & \multicolumn{3}{c}{ Age (years) } & \multicolumn{2}{c}{ Height $(\mathrm{cm})$} & \multicolumn{2}{c}{ Weight $(\mathrm{kg})$} \\
\cline { 2 - 8 } & Number & Mean & SD & Mean & SD & Mean & SD \\
\hline 4 to 4.5 years & 21 & 4.2 & 0.1 & 100.4 & 3.3 & 16.3 & 1.1 \\
4.5 to 5 years & 26 & 4.7 & 0.2 & 102.6 & 4.5 & 16.8 & 1.7 \\
5 to 5.5 years & 23 & 5.2 & 0.1 & 107.2 & 4.0 & 18.0 & 1.5 \\
5.5 to 6 years & 27 & 5.7 & 0.1 & 107.4 & 4.4 & 17.7 & 1.8 \\
6 to 6.5 years & 34 & 6.2 & 0.1 & 112.8 & 5.1 & 20.0 & 2.3 \\
6.5 to 7 years & 27 & 6.7 & 0.1 & 115.9 & 3.7 & 20.8 & 1.5 \\
\hline
\end{tabular}

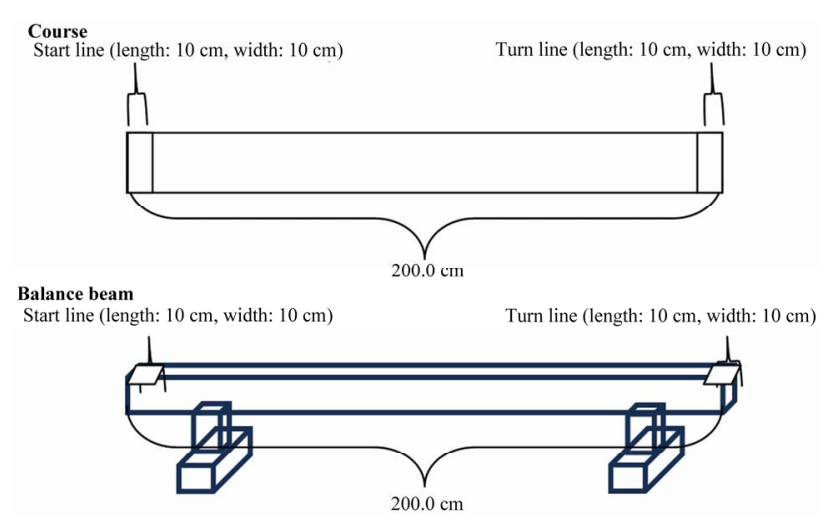

Figure 1.

Course illustration.

coefficients was tested. The level of significance was determined to be 0.05 .

\section{Results}

The ICC's of walking times for each condition were 0.61 0.81 for 4 -year-old boys, 0.64 - 0.83 for 5-year-old boys, and 0.66 - 0.79 for 6 -year-old boys.

Table 2 shows the basic statistics of walking times according to the test performed, age and obstacle condition, as well as the results of the three-way ANOVA. A significant interaction was found between age and test factors, between age and obstacle factors, and between test and obstacle factors. Multiple comparison showed that, in all age groups, the within-course walking time was shorter than the balance beam walking time. The former time was longer in boys aged 4 to 4.5 years than in 5 and 6 -year-old boys, and longer in boys aged 4.5 to 5 years than in boys aged 6.5 to 7 years. Balance beam walking time was longer in boys aged 4 to 4.5 years than in boys aged 4.5 to 5 years and in 5 and 6-year-old boys, longer in boys aged 4.5 to 5 years than in 5 and 6-year-old boys, and longer in 5-year-old boys than in 6-year-old boys. Under all conditions, the walking time was longer for 4-year-old boys than in 5 and 6-year-old boys, longer in boys aged 5 to 5.5 years than in 6-year-old boys, and longer in boys aged 5.5 to 6 years than boys aged 6.5 to 7 years. In addition, the within-course walking time was shorter than the balance beam walking time under all conditions. Both walking times were shorter in the following order: no obstacle (shortest), low obstacle, and high obstacle (longest).

Figure 2 shows regression coefficients of each within-course walking time. The no obstacle and low obstacle conditions showed 
Table 2.

The basic statistics of walking times according to test performed, age, and obstacle condition, and results of the three-way ANOVA.

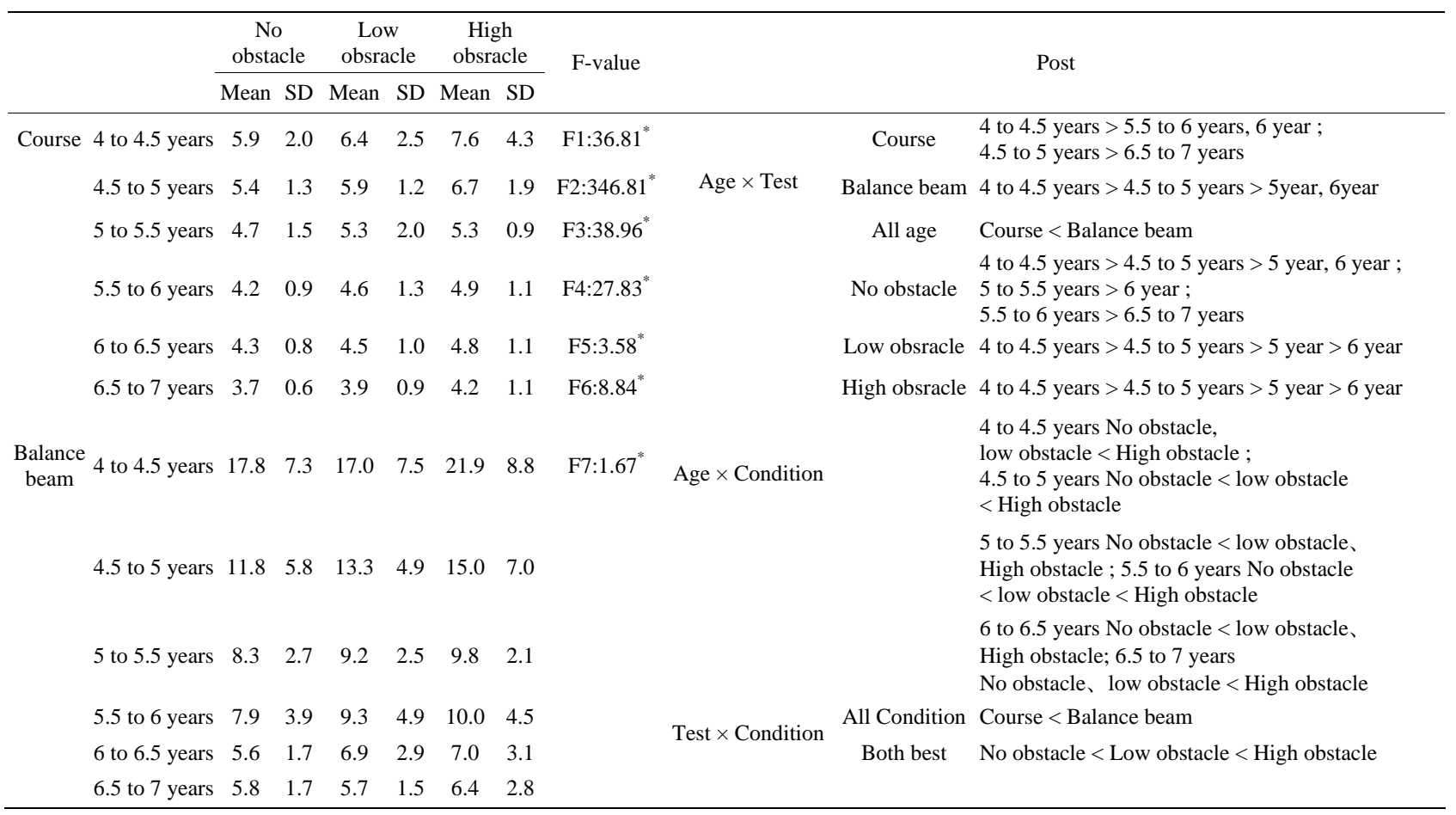

${ }^{*} p<0.05$, Unit: sec, F1: Age; F2: Test; F3: Condition; F4: Interaction (Age $\times$ Test); F5: Interaction (Age $\times$ Condition); F6: Interaction (Test $\times$ Condition); F7: Interaction $($ Age $\times$ Test $\times$ Condition $)$. F1 $(5,149)=36.82$, F2 $(1,149)=346.79$, F3 $(2,298)=39.04$, F4 $(5,149)=27.38$, F5 $(10,298)=3.55$, F6 $(2,298)=8.87$, F7 $(10,298)=1.68$

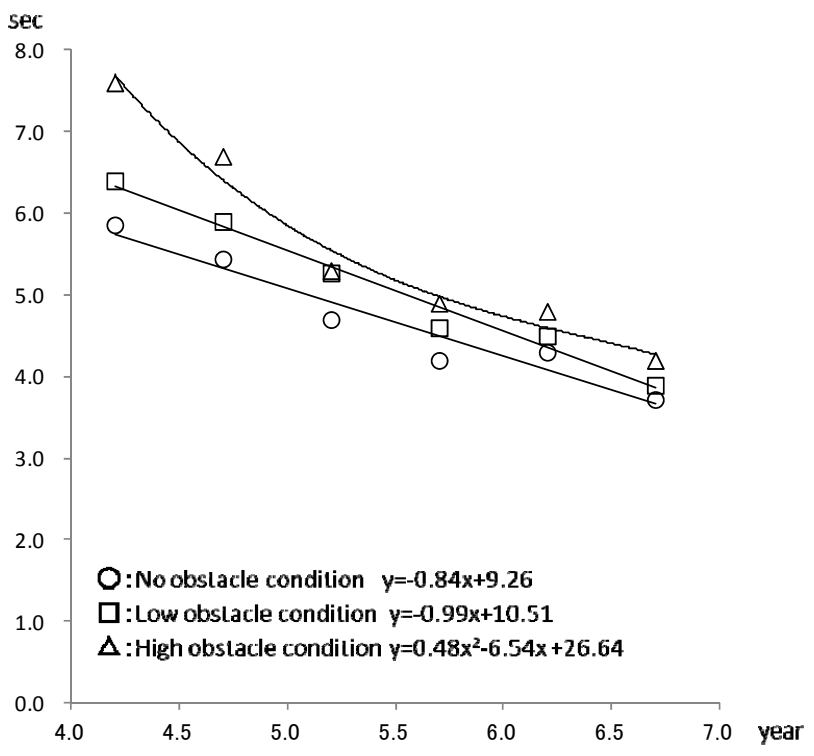

Figure 2.

Regression coefficients for each course walking time.

a linear regression and the high obstacle condition showed a curvilinear regression. Figure 3 shows regression coefficients for each balance beam walking time. A curvilinear regression was found under all conditions.

\section{Discussion}

The within-course walking time was faster than the balance

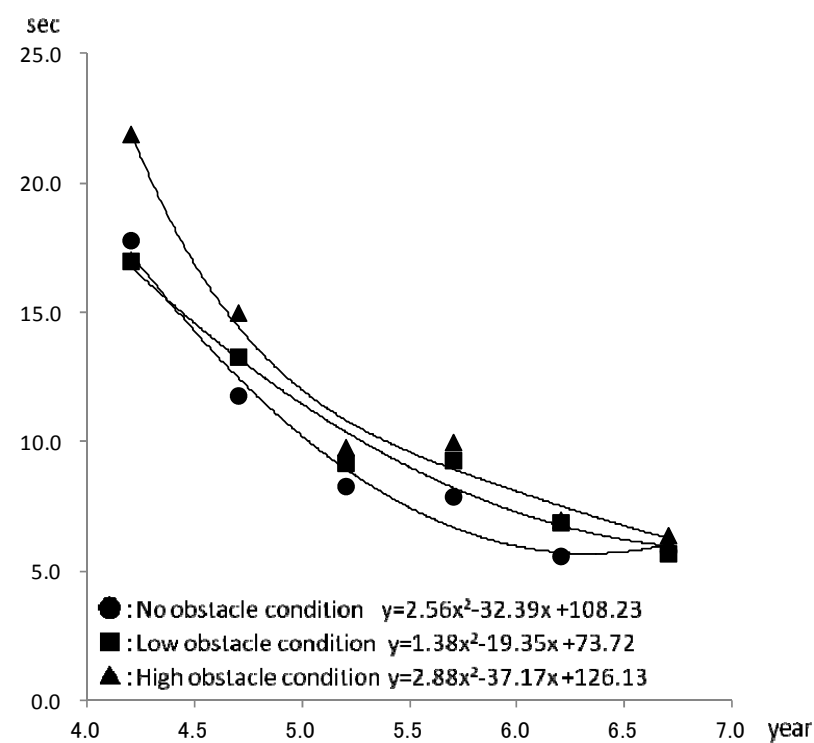

Figure 3.

Regression coefficients for each balance beam walking time.

beam walking for all age groups. In order to complete the test on the balance beam, a change in physical direction on a path that is high and narrow is necessary. Because walking on a balance beam always places the participant in an unstable posture state, preschool children may find it very difficult. It is judged that the ability to complete the balance beam walking test, as compared with level walking (Aoki et al., 2011), takes more time due to the fact that it is more difficult. In addition, 
regardless of the obstacle setting, the within-course walking time was slower for 4-year-old boys than for 5 and 6-year-old boys, and balance beam walking was slower for 5-year-old boys than for 6-year-old boys. Demura et al., (1994) examined age differences in line walking time using children aged 3 to 6 years old, and reported that it shortens with age. According to Kasuga et al., (in press), the within-course walking time was longer for 4-year-old children than for 5 and 6-year-old children. On the other hand, Clifton (1978) reported that the balance beam walking test score rises with age. Demura et al., (1994) also reported in the above-stated study that balance beam walking time shortens with age. According to Harcherik et al., (1982) report, balance beam walking speed tends to become faster with age, with 4 - 5 years old group the slowest, the 6 - 8 years old group faster, and the 9 - 11 years old group fastest, but showed insignificant difference between the 9 - 11 years old and 12 - 14 years old age groups. Demura (1995) reported that dynamic balance develops steadily from about 4 years old, since balance beam walking becomes faster between the ages of 3.5 - 6.5 years old. Children who develop early can walk at about 1 year and run at about 2 years of age. According to Aoki et al., (2011), children over the age of 6 can step over an obstacle smoothly. It is considered that in-course and balance beam walking can both be performed faster by 5 year olds than by 4 year olds, and faster by 6 years old than by 5 years old due to an an increase of walking frequency in addition to development of basic movement ability with age.

On the other hand, it was confirmed that even for withincourse walking, walking time with a low obstacle (for which difficulty is low) decreases proportionally with age, but both within-course walking in the presence of a high obstacle and balance beam walking (for which difficulty is high) show little reduction in walking time after 5 years in age. Within-course walking is an easy movement for preschool children over 3 years who can walk smoothly. Takagi (2009) reported that even preschool children were able to perform a few movements simultaneously (for example, throw a ball while walking) through age 6 . It is inferred that they can change from simple movements i.e., walking, to compound movements with relatively high difficulty, such as stepping over an obstacle while walking. The mean time presented above of within-course walking (no obstacle condition) was 5.9 seconds for boys aged 4 to 4.5 years, and 3.7 second for boys aged 6.5 to 7 years, and their time difference was about 2.2 seconds. In contrast, the mean of balance beam walking time (no obstacle condition), a high difficulty task, was 17.8 seconds for boys aged 4 to 4.5 years, and 5.8 second for boys aged 6.5 to 7 years, and their time difference was 12.0 seconds. In summary, the balance beam walking times with high difficulty were about six times longer $(12.0 / 2.2=5.5)$ than the in-course walking times. In addition, differences between the within-course walking times and the balance beam walking times for each obstacle condition ranged 10.6 - 16.0 seconds for boys aged 4 to 4.5 years, but ranged from 3.9-5.8 seconds for 5-year-olds and ranged from 1.8 - 2.7 seconds for 6 -year-olds. Essentially, the difference was large between boys aged 4 to 4.5 years and 5 to 6 years.

From the above, it is judged that when adding a balance beam or an obstacle to the walking path, younger children are more greatly affected than children over 5 years old. Older children can achieve certain walking times through improving the ability to perform compound movements in addition to improvements in walking ability with age, even if the difficulty of the movement task is high. Hence, it is considered that children aged 5.5 to 6 years can even accomplish very difficult movements such as stepping over a $10 \mathrm{~cm}$ obstacle or walking on the balance beam. On the other hand, development of dynamic balance with age may differ according to the type and difficulty of the test. However, this problem has not been well examined. This study clarifies that development of walking time in children 4 - 6 years old differs by type of test and difficulty of movement tasks. We will need use tests and movement tasks appropriate to each age level in order to evaluate dynamic balance of preschool children.

Both the within-course walking and balance beam walking times were slower in the following order: no obstacle (fastest), low obstacle, and high obstacle (slowest). When stepping over an obstacle, children must lift one foot over the obstacle (Aoki et al., 2011). It is necessary to maintain a stable posture by supporting one leg to lift it over a tall object.

In addition, greater exertion of dynamic balance is required to lift one leg very high during walking (Kasuga et al., in press). As an obstacle set on a walking course becomes higher, it takes more time to step over, causing the contribution of dynamic balance to become larger. Hence, it is inferred that the effect of stepping over an obstacle while walking becomes larger as it becomes higher for both in-course and balance beam walking.

In this study, due to subjects' limited walking ability, a narrow course (10 cm width) and balance beam or an obstacle was used. The results of this study describe that although walking time shortens remarkably with age until 5.5 years in both walking with high obstacle and balance beam walking, it changes little after 5.5 years old. It is thus indicated that dynamic balance related to walking develops until age 6 , when children of this age stage are able to perform plural exercise tasks simultaneously with comparative ease. The findings on development of dynamic balance obtained in this study are considered to be very useful when considering age level in teaching exercise tasks to preschool children.

\section{Conclusion}

In conclusion, preschool children can walk faster withincourse than on a balance beam regardless of the presence of an obstacle or their age. Development of the ability to perform both walking tasks with age may differ.

\section{REFERENCES}

Aoki, H., Demura, S., Kasuga, K., Shin, S., \& Kawabata, H. (2011). Examining difference in walking time on a balance beam with an obstacle based on gender and age in preschool children. Japanese Society of Education and Health Science, 56, 352-355.

Bürgi, F., Meyer, U., Granacher, U., Schindler, C., Marques-Vidal, P., Kriemler, S., \& Puder, J. J. (2011). Relationship of physical activity with motor skills, aerobic fitness and body fat in preschool children: a cross-sectional and longitudinal study (Ballabeina). International Journal of Obesity, 35, 937-944. doi:10.1038/ijo.2011.54

Chandler, L. S., Richardson, G. A., Gallagher, J. D., \& Day, N. L. (1996). Prenatal exposure to alcohol and marijuana: effects on motor development of preschool children. Alcoholism: Clinical \& Experimental Research, 20, 455-461.

doi:10.1111/j.1530-0277.1996.tb01075.x

Clifton, M. A. (1978). Effects of special instruction and practice by preschool age children on performance of object projection and stability tests. Percept Mot Skills, 47, 1135-1140.

doi:10.2466/pms.1978.47.3f.1135 
Demura, S. (1993). Contribution of physical fitness and throw form to ball-throw distance and the sex difference in preschool children. Japan Journal of Physics Education and Health Sports Science, 37, 339-350.

Demura, S., Nagasawa, Y., \& Kasuga, K. (1994). The development of dynamic balance and its sex difference in preschool children. Journal of Educational Medicine, 39, 368-376.

Demura, S. (1995). Development and sexual difference of static and dynamic balance in preschool children. Japan Journal of Physics Education, 40, 67-79.
Harcherik, D. F., CArbonari, C. M., \& Cohen, D. J. (1982). Attentional and perceptual measures: Developmental changes. Schizophrenia Bulletin, 8, 349-355.

Kasuga, K., Demura, S., Aoki, H., Shin, S., Sugiura, H., \& Uchida, Y. (in press). Sex and age-level differences of walking time in preschool children on an obstacle course. Journal of Physiological Anthropology.

Takagi, N. (2009). Exercise play by preschool children (latest ed.). Tokyo: Fumaidou-Syuppan, 50-64. 